\title{
Increased mean arterial pressure response to dynamic exercise in normotensive subjects with multiple metabolic risk factors
}

\author{
Nobuyuki Miyai ${ }^{1}$, Maki Shiozaki $^{2}$, Mayuko Yabu ${ }^{1}$, Miyoko Utsumi ${ }^{1}$, Ikuharu Morioka ${ }^{1}$, Kazuhisa Miyashita $^{2}$ \\ and Mikio Arita ${ }^{1}$
}

Metabolic syndrome (MS) may influence vascular reactivity and might cause an excessive increase in blood pressure (BP) during dynamic exercise. We examined this hypothesis in 698 normotensive men (mean age: 43 years) free of cardiovascular disease, diabetes mellitus and renal disease. The response of BP to exercise was assessed by the mean arterial pressure (MAP) during bicycle ergometry. The MAP values were expressed as $z$-scores normalized to the relative increases in heart rate. High-normal BP, dyslipidemia and hyperglycemia were diagnosed according to the Japan-specific MS criteria. The $z$-score of MAP was significantly higher in subjects with high-normal $\mathrm{BP}(+0.57, P<0.001)$, dyslipidemia $(+0.18, P<0.001)$ and hyperglycemia $(+0.24, P<0.001)$ than in those without MS component $(-0.38)$. In the high-normal BP subjects, the addition of dyslipidemia and/or hyperglycemia was associated with a progressive increase in the $z$-score of exercise MAP, whereas no such association was observed in the normal-BP subjects $(P=0.033$, two-way ANOVA). Multivariate regression analysis revealed that a greater number of MS components $(\beta=0.102, P=0.010)$ was an independent determinant of increased MAP $z$-score after adjustment for potential confounders, including age $(\beta=0.123, P<0.001)$, body mass index $(\beta=0.145, P<0.001)$ and high-normal BP $(\beta=0.410, P<0.001)$. These results suggest that accumulation of MS components may alter vascular structure and function and lead to the significant elevation of MAP during dynamic exercise even before clinical manifestation of arterial hypertension.

Hypertension Research (2013) 36, 534-539; doi:10.1038/hr.2012.215; published online 24 January 2013

Keywords: exercise; hypertension; mean arterial pressure; metabolic risk factors

\section{INTRODUCTION}

An exaggerated response of arterial blood pressure (BP) to exercise is an independent predictor of future hypertension ${ }^{1-3}$ and is associated with greater risk of end-organ hypertrophy, ${ }^{4}$ stroke $^{5}$ and cardiovascular disease mortality. ${ }^{6,7}$ Although the mechanisms linking aberrant BP elevation during exercise and adverse outcome are not yet completely understood, recent studies in various clinical settings have indicated that pathophysiological changes and its complications are associated with an exaggerated response of BP to exercise even in the early stages of arterial hypertension. ${ }^{8-14}$

The metabolic syndrome (MS) consists of multiple, interrelated risk factors of metabolic origin that appear to promote the development of atherosclerotic cardiovascular diseases in a direct manner. ${ }^{15}$ The constellation of metabolic factors may also lead to hypertension and its complications via structural and functional alterations to the vascular wall. Such an effect might have a greater influence on vascular reactivity than on basal tone. ${ }^{16-19}$ Therefore, although resting BP may not necessarily be high when the vascular impairment is not yet very severe, the vascular reactivity may already be abnormal, possibly resulting in excessive BP elevation under stressful conditions, for example, during physical exertion.

Therefore, the objective of this study was to identify the effects of clustering of metabolic risk factors on arterial BP response to dynamic exercise in apparently healthy, normotensive individuals.

\section{METHODS}

Study population

A cross-sectional sample of 698 healthy normotensive men (age range: 20-59 years) was recruited for the study from a group who submitted to bicycle ergometry testing in combination with medical examinations conducted at our laboratory. Subjects were enrolled if their resting BP measurements in the absence of antihypertensive medication were confirmed to be consistently lower than 140/90 mm Hg on two separate occasions. Subjects with a history of cerebro-cardiovascular disease, renal disease, diabetes mellitus, syndromal obesity or thyropathy, as well as those with electrocardiographic evidence of coronary heart disease or cardiac arrhythmia, were excluded from the present study. Any individuals who engaged in systematic physical training, that was

${ }^{1}$ School of Health and Nursing Science, Wakayama Medical University, Wakayama, Japan and ${ }^{2}$ Department of Hygiene, School of Medicine, Wakayama Medical University, Wakayama, Japan

Correspondence: Dr N Miyai, School of Health and Nursing Science, Wakayama Medical University, P.0. Box 641-0011, 580 Mikazura, Wakayama 641-0011, Japan. E-mail: miyain@wakayama-med.ac.jp

Received 19 June 2012; revised 18 November 2012; accepted 19 November 2012; published online 24 January 2013 
planned, structured and repetitive for the purpose of conditioning any part of the body, were not included, and in most cases, the subjects were sedentary or performed only light activity at work and at home. Also excluded were individuals who were unable to reach the submaximal workload of $100 \mathrm{~W}$ during exercise test on a bicycle ergometer. Written informed consent to participate and clearance for the examination data to be used were obtained from the subjects after they had received an explanation of the study aim and procedures. The protocol was approved by the Ethics Committee of Wakayama Medical University.

\section{General procedure}

Subjects underwent general laboratory examinations including anthropometric, physiological and blood biochemical measurements. All measurements were carried out in a room that was quiet and temperature-controlled and were conducted in the morning after an overnight fast and abstinence from smoking and caffeine for at least $3 \mathrm{~h}$ before the examination. In addition, any subjects taking medications were advised to refrain from taking them for $24 \mathrm{~h}$ before the examination.

The anthropometric measurements were collected with each subject wearing light clothing without shoes. Body mass index (BMI) was calculated as the weight-to-squared-height ratio $\left(\mathrm{w} / \mathrm{h}^{2}\right)$. The subjects were allowed to rest for at least $5 \mathrm{~min}$, and then brachial arterial BP was measured three times on the left arm with the subjects in the sitting position by well-trained physicians using a mercury sphygmomanometer. The last two readings were averaged to obtain the representative values for systolic BP (SBP) and diastolic BP (DBP). After the resting $\mathrm{BP}$ measurements, the subjects were subjected to an exercise tolerance test using a bicycle ergometer to measure the $\mathrm{BP}$ and heart rate (HR) during dynamic physical exertion. A fasting blood sample was drawn from the antecubital vein for the measurement of lipid and glucose profiles by standard techniques.

\section{Bicycle ergometry test}

A symptom-limited, graded exercise test was performed on a bicycle ergometer (Fukuda Denshi: ML-1400, Tokyo, Japan). The subjects rested seated for $5 \mathrm{~min}$ to establish baseline readings and then cycled until they complained of exhaustion. The workload was progressively increased using a linear-slope method at a rate of $12.5 \mathrm{~W}$ per min. ${ }^{3}$ Electrocardiogram lead $\mathrm{V}_{3}$ and $\mathrm{HR}$ were monitored continuously throughout the test period, and BP was recorded noninvasively every minute using an automated sphygmomanometer (Nippon Colin: STBP-780B, Komaki, Japan) with an appropriately sized cuff. The device was an auscultatory unit using R-wave gating to identify Korotkoff sounds and was proven previously to be reliable and its results reproducible. ${ }^{20}$

\section{Data analysis}

The response of BP to exercise was evaluated based on the mean arterial pressure (MAP) during submaximal exercise at a workload of $100 \mathrm{~W}$. The MAP was calculated from the following equation: $\mathrm{MAP}=\mathrm{DBP}+(\mathrm{SBP}-\mathrm{DBP}) / 3$. The HR was used as the index of physiological exercise intensity and was expressed as a percentage of the maximal HR reserve. The maximal HR reserve was calculated from the following formula: (HR at $100 \mathrm{~W}$ of exercise -resting $\mathrm{HR}) /($ age-specific maximal $\mathrm{HR}$-resting $\mathrm{HR}) \times 100{ }^{21}$ The relationship between MAP and HR increment was analyzed for each individual subject. The mean and standard deviation (s.d.) of MAP were then calculated for six subgroups defined by the percent increase in HR $(0-39 \%, 40-49 \%, 50-59 \%$, $60-69 \%, 70-79 \%$ and $80-100 \%)$. From these normal data, a $z$-score of exercise MAP was calculated for each subject. The mean value of the $z$-score in all subjects was 0 with a s.d. of 1 . The $z$-score results are presented as the mean ( $95 \%$ confidence interval). The metabolic risk profiles of the individuals were determined based on the Japan-specific criteria for diagnosis of MS. ${ }^{22}$ A subject was considered to have high-normal BP if he had systolic BP $\geqslant 130 \mathrm{~mm} \mathrm{Hg}$ or diastolic BP $\geqslant 85 \mathrm{~mm} \mathrm{Hg}$ or used antihypertensive medication, dyslipidemia if he had a high-density lipoprotein (HDL) cholesterol $<1.03 \mathrm{moll}^{-1}$ or total triglycerides (TG) $\geqslant 1.7 \mathrm{moll}^{-1}$ or used antidyslipidemia medication and hyperglycemia if he had fasting blood glucose $(\mathrm{FBG}) \geqslant 6.1 \mathrm{moll}^{-1}$ or used hypoglycemic medication.
The results are expressed as the mean \pm s.d. for continuous variables and percentage for categorical variables, unless otherwise specified. If a variable was not normally distributed, then logarithmic transformation was performed before regression analysis. Univariate and multivariate regression analyses were performed to evaluate the correlations of the BP response to exercise with baseline clinical and exercise testing parameters. Differences in outcome parameters according to the presence and absence of a MS component were tested with Student's $t$-tests for unpaired values. The effects of the clustering of dyslipidemia and/or hyperglycemia in high-normal-BP subjects on exercise MAP were compared with those of normal-BP subjects using two-way ANOVA (group (high-normal vs normal) $\times$ number of MS components (none vs either vs both). When appropriate, post-hoc testing was performed using one-way ANOVA followed by the Bonferroni correction for multiple comparisons. Multivariate regression analysis was performed to estimate the strength and independence of the effect of a greater number of MS components on the BP response to exercise. The following parameters were entered into the stepwise multiple regression model as independent variables: age, BMI, current smoking, SBP, DBP, HR, TG, total cholesterol, HDL cholesterol, FBG, high-normal BP, dyslipidemia, hyperglycemia and number of MS components. The null hypothesis was rejected at a level of significance of $P<0.05$, and all statistical tests were two-tailed. Data analyses were performed using the SPSS statistical package (SPSS Software, Inc., Chicago, IL, USA).

\section{RESULTS}

The mean age of the study population was 43 years. Smokers, defined as those currently smoking greater than one cigarette per day, made up $43 \%$ (Table 1). The prevalence of overweight (preobese) was $19.9 \%$ and the prevalence of obesity (class I) $2.3 \%$. According to the Japan-specific MS criteria, the population included subjects with high-normal BP $(n=264,37.8 \%)$, dyslipidemia $(n=259,37.1 \%)$ and hyperglycemia $(n=190,27.2 \%)$. Subjects with normal BP $(n=434)$ were significantly less likely than those with high-normal BP to have dyslipidemia (33.9\% vs $42.8 \%, P=0.018)$ or hyperglycemia $(23.3 \%$ vs $33.7 \%, P=0.003)$. During the bicycle ergometry test, the mean SBP, DBP and MAP at the workload of $100 \mathrm{~W}$ were $172 \mathrm{~mm} \mathrm{Hg}, 88 \mathrm{~mm} \mathrm{Hg}$ and $116 \mathrm{~mm} \mathrm{Hg}$, respectively. HR progressively increased to a mean of 129 beats per min, corresponding to approximately $50 \%$ of maximal HR reserve.

The exercise MAP correlated positively with age, BMI, resting SBP and DBP, MAP, HR, TG, total cholesterol and FBG and negatively with HDL-cholesterol (Table 1). Furthermore, higher MAP during exercise was closely associated with a greater increase in HR and lower cardiorespiratory fitness, which was assessed by the estimated maximum oxygen uptake or physical working capacity. Multiple stepwise regression analysis revealed that age, SBP, DBP, MAP, maximal HR reserve and maximum oxygen uptake were significant determinants of the MAP during exercise. The percent increase in HR during exercise was strongly correlated with the MAP and was identified by multivariate analysis as the most important determinant. The MAP values were therefore normalized to the percent increase in HR and expressed as $z$-scores in order to allow comparisons of MAP during exercise at the same physiological exercise intensity in individuals with varying levels of physical capacity (Figure 1).

The MAP during exercise was significantly higher in the subjects with high-normal resting BP than in those with normal BP ( $z$-score: +0.58 vs $-0.34, P<0.001$; Figure 2$)$. Furthermore, the MAP was significantly higher in subjects with dyslipidemia $(z$-score: +0.18 vs $-0.10, P<0.01)$ or hyperglycemia $(z$-score: +0.24 vs -0.08 , $P<0.01)$ than in those without such MS component factors. We divided the high-normal-BP subjects according to the number of additional MS components (dyslipidemia and hyperglycemia) 
Table 1 Clinical characteristics and exercise testing data of the study subjects as well as univariate and multivariate analyses of the variables associated with mean arterial pressure during exercise

\begin{tabular}{|c|c|c|c|c|c|}
\hline \multirow[b]{2}{*}{ Variables } & & \multicolumn{2}{|c|}{ Univariate analysis } & \multicolumn{2}{|c|}{ Multiple linear regression analysis ${ }^{\mathrm{a}}$} \\
\hline & & r & $\mathrm{P}$ & $\beta$ & $\mathrm{P}$ \\
\hline \multicolumn{6}{|l|}{ Baseline measurements } \\
\hline Body mass index $\left(\mathrm{kg} \mathrm{m}^{-2}\right)$ & $22.8 \pm 2.6$ & 0.062 & 0.049 & 0.102 & 0.001 \\
\hline Systolic blood pressure $(\mathrm{mm} \mathrm{Hg})$ & $125.2 \pm 8.6$ & 0.410 & $<0.001$ & 0.260 & $<0.001$ \\
\hline Heart rate (beats/minute) & $75.6 \pm 11.4$ & 0.142 & $<0.001$ & & \\
\hline Triglycerides $\left(\mathrm{moll}^{-1}\right)^{\mathrm{b}}$ & $1.50 \pm 0.71$ & 0.162 & $<0.001$ & & \\
\hline Total cholesterol $\left(\mathrm{moll}^{-1}\right)$ & $5.10 \pm 0.85$ & 0.155 & $<0.001$ & & \\
\hline HDL cholesterol $\left(\mathrm{moll}^{-1}\right)$ & $1.37 \pm 0.34$ & -0.102 & 0.004 & & \\
\hline Fasting glucose $\left(\mathrm{moll}^{-1}\right)$ & $5.25 \pm 0.82$ & 0.179 & $<0.001$ & & \\
\hline Current smoker (\%) & 42.9 & & & & \\
\hline \multicolumn{6}{|l|}{ Exercise testing data } \\
\hline Diastolic blood pressure $(\mathrm{mm} \mathrm{Hg})$ & $88.2 \pm 10.3$ & & & & \\
\hline Mean arterial pressure $(\mathrm{mm} \mathrm{Hg})$ & $116.4 \pm 12.4$ & & & & \\
\hline Heart rate (beats per min) & $128.7 \pm 14.9$ & 0.424 & $<0.001$ & & \\
\hline Maximum heart rate reserve (\%) & $51.7 \pm 12.7$ & 0.493 & $<0.001$ & 0.421 & $<0.001$ \\
\hline Maximum oxygen uptake (I per min) & $2.43 \pm 0.31$ & -0.428 & $<0.001$ & -0.117 & 0.003 \\
\hline Physical working capacity (Watt) & $106.1 \pm 22.8$ & -0.475 & $<0.001$ & & \\
\hline
\end{tabular}

Values are mean \pm s.d. and percentage.

$r$ indicates correlation coefficient; $\beta$, standardized partial regression coefficient.

avalues considered in the multiple regression fasting model were age, body mass index, resting systolic and diastolic blood pressure, maximal heart rate reserve, physical working capacity, maximal oxygen uptake, triglycerides, total cholesterol, high density lipoprotein cholesterol, and fasting glucose. Adjusted model $R^{2}=0.498, \mathrm{~F}$ ratio $=110.43$, model probability $P<0.001$.

oxygen uptake, triglycerides, total cholesterol, high density lipoprotein cho

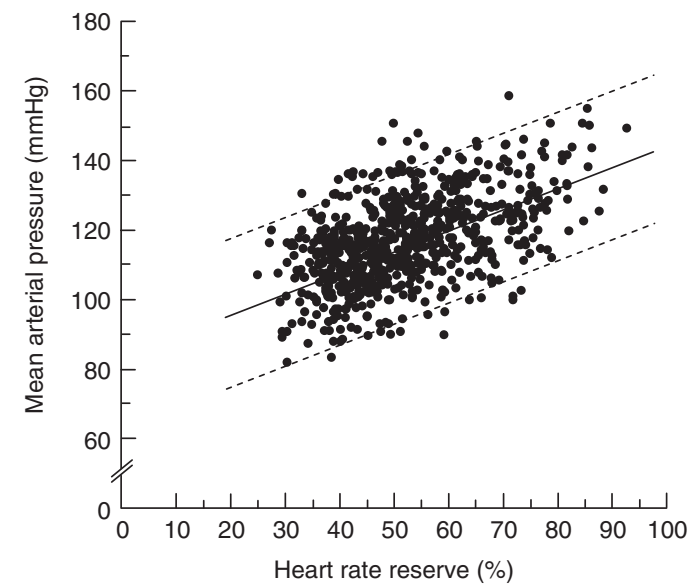

Figure 1 Scatter plot of mean arterial pressure vs percentage of heart rate reserve. The lines represent the mean (solid line) and the $95 \%$ prediction interval (dashed line). $r=0.493(P<0.001)$.

present; 104 (39.4\%) had no additional MS component, 118 (44.7\%) had one additional MS components and 42 (15.9\%) had both additional MS components. Although these subgroups had statistically different but clinically similar resting MAP values $(98 \mathrm{~mm} \mathrm{Hg}$, $100 \mathrm{~mm} \mathrm{Hg}$ and $100 \mathrm{~mm} \mathrm{Hg} ; P<0.020$, ANOVA), the presence of dyslipidemia and/or hyperglycemia was associated with significantly
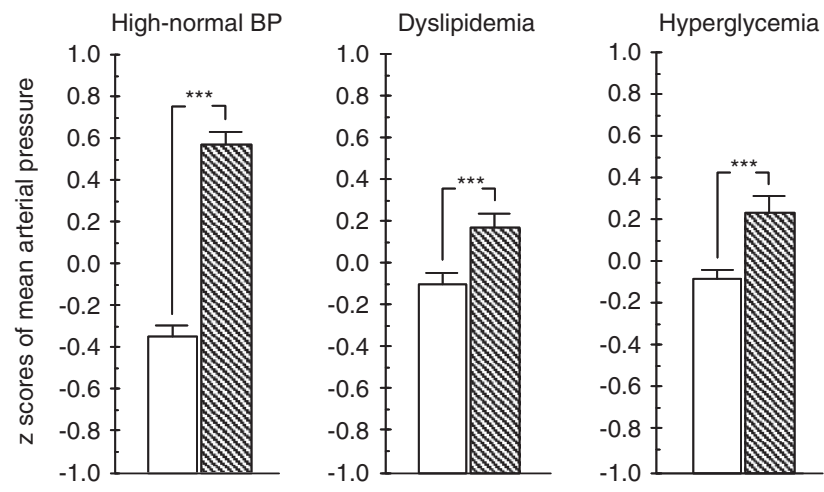

Figure 2 The bars represent the $z$-scores of mean arterial pressure during exercise according to the single dichotomized components of the metabolic syndrome. Striped bars indicate subjects with high-normal BP $(n=264)$, dyslipidemia $(n=259)$, and hyperglycemia $(n=190)$. Data are shown as the mean \pm s.e.m. ${ }^{* *} P<0.001$ by Student's $t$-test.

increased MAP during exercise $(z$-score: $+0.33,+0.62$ and +1.05 ; $P<0.001$; Figure 3). This dose-responsive increase in MAP was not clearly present in subjects with normal BP $(z$-score: $-0.38,-0.32$ and $-0.20 ; P=0.453)$. The group effect $(P<0.001)$, MS component effect $(P<0.001)$, and group $\times$ MS component interaction effect $(P=0.033)$ were significant by two-way ANOVA. 


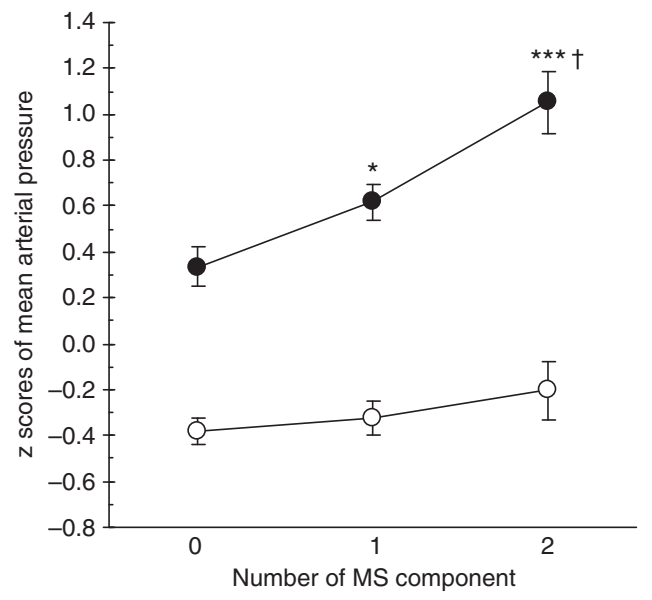

Figure 3 The circle plots represent the behaviors of the z-scores of mean arterial pressure during exercise in the high-normal-BP and normal-BP subjects with increasing numbers of metabolic syndrome components (presence or absence of dyslipidemia and/or hyperglycemia). Closed circles indicate high-normal-BP subjects and open circles normal-BP subjects. Data are shown as the mean \pm s.e.m. The group effect $(P<0.001)$, MS component effect $(P<0.001)$ and group $\times$ MS component interaction effect $(P=0.033)$ were significant by two-way ANOVA. ${ }^{*} P<0.05$, ${ }^{* * *} P<0.001$ vs the subjects without any MS component; $\stackrel{\dagger}{P}<0.05$ vs the subjects with $1 \mathrm{MS}$ component by post-hoc analysis using the Bonferroni correction.

Table 2 Multiple regression analysis to assess the independent associations between the z-score of mean arterial pressure during exercise and metabolic risk parameters

\begin{tabular}{|c|c|c|c|c|c|}
\hline Variables ${ }^{a}$ & $\begin{array}{l}\text { Regression } \\
\text { coefficient }\end{array}$ & $S E$ & $\beta$ & $\mathrm{t}$ & $\mathrm{P}$ value \\
\hline Resting high-normal BP & 0.844 & 0.069 & 0.410 & 12.188 & $<0.001$ \\
\hline BMI $\left(\mathrm{kg} \mathrm{m}^{-2}\right)$ & 0.056 & 0.013 & 0.145 & 4.199 & $<0.001$ \\
\hline Age (years) & 0.015 & 0.004 & 0.123 & 3.682 & $<0.001$ \\
\hline $\mathrm{N}$ of MS component & 0.104 & 0.050 & 0.102 & 2.085 & 0.010 \\
\hline (Constant) & -2.310 & 0.346 & - & -6.679 & $<0.001$ \\
\hline
\end{tabular}

Adjusted $R^{2}, 0.247 ; F$-value, 56.84; model probability $P<0.001$.

$\mathrm{SE}$ indicates standard error; $\beta$, standardized partial regression coefficient; BMI, body mass index; N of MS component, additional number of metabolic syndrome components (dyslipidemia and/or hyperglycemia).

Values considered in the multiple regression model were age, body mass index, smoking systolic and diastolic blood pressures, heart rate, triglycerides, total cholesterol, high-density lipoprotein cholesterol, fasting glucose, high-normal BP, dyslipidemia, hyperglycemia and number of MS components.

Multivariate regression analysis revealed that a greater number of MS components was an independent determinant of increased MAP during exercise after adjustment for potential confounders including age, BMI and resting high-normal BP (Table 2). Moreover, when dyslipidemia and hyperglycemia were isolatedly entered into the regression model, instead of the number of MS components, hyperglycemia but not dyslipidemia was selected as a significant determinant of exercise MAP $(\beta=0.076, P<0.032)$.

\section{DISCUSSION}

This cross-sectional study of a population-based sample of apparently healthy men clearly demonstrates that clustering of MS components, including high-normal BP, dyslipidemia and hyperglycemia, is associated with an excessive increase in MAP during exercise stress testing even in the absence of clinical hypertension. The observation suggests that subjects with multiple metabolic risk factors likely have subtle vascular abnormalities, leading to the impairment of the peripheral vasodilatory response to dynamic exercise. The findings also indicate that such an effect may have a greater influence on vascular reactivity than on basal tone; therefore, it can be speculated that elevation of MAP during dynamic exercise might unmask preclinical atherosclerotic changes caused by the constellation of risk factors.

An excessive increase in BP during exercise has been found to predict a future diagnosis of hypertension, ${ }^{1-3}$ end-organ hypertrophy, ${ }^{4}$ and cerebro-cardiovascular disease mortality. ${ }^{5-7}$ Recent studies have indicated the vascular abnormalities of patients with excessive increases in BP during exercise, such as reduced endothelium-dependent vasodilatation capacity, ${ }^{8-10}$ increased arterial stiffness ${ }^{10-12}$ and increased prevalence of carotid atherosclerosis. ${ }^{13}$ On the basis of these findings, our study focused on the effects of the accumulation of MS components on the response of $\mathrm{BP}$ to dynamic exercise. In the subjects with high-normal BP, the addition of dyslipidemia and/or hyperglycemia was associated with a significantly elevated MAP ( $z$-score) during dynamic exercise, and this elevation increased with an increasing number of MS components. Furthermore, multivariate regression analysis revealed that a greater number of MS components was an independent determinant of high MAP during exercise after adjustment for potential confounders, including age and BMI. These findings are in agreement with previous published reports of significant associations with high lipoprotein cholesterol, ${ }^{10,16}$ impaired glucose metabolism, ${ }^{16,19}$ and $\mathrm{MS}^{18}$ with exercise BP. In addition, the present results indicate that the accumulation of risk factors is also an important predictor of $\mathrm{BP}$ response to exercise.

The finding of an excessive increase in BP during exercise in association with multiple metabolic risk factors despite the absence of arterial hypertension might be explained by the existence of structural and functional impairments of the vasculature, which alter its ability to respond to physical stress. During dynamic exercise, cardiac output increases in response to the demand of working muscle. The BP response to the increase in cardiac output is moderated by a decrease in peripheral vascular resistance via peripheral vasodilatation. This is partly attributable to an enhanced release of nitric oxide during exercise due to vascular wall shear stress. ${ }^{23}$ It is likely that endothelial dysfunction limits the peripheral vasodilatation that normally buffers against an excessive increase in BP during dynamic exercise. The accumulation of MS component factors may alter vascular structure and function and thus limit exercise-induced endothelial vasodilatation, thereby contributing to the significant elevation of the BP in response to exercise even in the absence of arterial hypertension. In this regard, a previous study of large populationbased sample ${ }^{10}$ demonstrated that cardiovascular risk factors were important predictors of exercise BP as well as that abnormal vascular function (as assessed by arterial stiffness and endothelial function) was a contributing factor in the exaggerated BP response to exercise.

In agreement with previous studies, ${ }^{1,3,10,18,24}$ an excessive increase in MAP during exercise is significantly related to age after adjustment for the other explanatory factors. Age is associated with alterations in a number of structural and functional properties of the arteries, including wall thickness, wall stiffness and endothelial dysfunction. Arterial stiffening with advancing age reduces the ability of the arterial tree to buffer the increased arterial pressure generated by left 
ventricular ejection. ${ }^{25}$ Such an effect contributes to the exaggeration of the response of BP to exercise and, further, induces subsequent resting hypertension. In addition, BMI significantly influenced the elevation of BP during exercise. Excess weight is well known to be associated with increased peripheral vascular resistance; therefore, many studies have shown a directly proportional relationship between weight gain and excessive response of BP to exercise. ${ }^{10,18}$

Our studies revealed that dyslipidemia and hyperglycemia in conjunction with high-normal BP were associated with greater BP elevation, whereas some other studies have failed to find such relationships. ${ }^{2,18}$ These discrepant results may arise partly from the different exercise intensities at which the exaggerated responses of BP were determined. We evaluated the response of BP to exercise based on the data obtained during a submaximal level of bicycle ergometry testing (that is, at a workload of $100 \mathrm{~W}$ ). The HRs of the study subjects attained about $50 \%$ of their maximal HR reserve values, which was considered to correspond to the anaerobic threshold. The sympathetic nervous and renin-angiotensin systems are highly activated during exercise above the AT, and hence the plasma levels of vasoconstrictive substances, such as catecholamines, angiotensin II and endothelin-1, increase exponentially. ${ }^{26,27}$ Although many previous studies have evaluated the peak BP in response to maximal exercise, it is speculated that monitoring of the $\mathrm{BP}$ response to a relatively low, sub-maximal workload is preferable for assessing the vasodilatory response to exercise. ${ }^{10,16,23}$ The effects of the metabolic risk factors on vascular reactivity might be more clearly identified during mild to moderate exercise than during maximal exercise.

As the other possible explanation, although a number of $\mathrm{BP}$ parameters are available, we used MAP rather than SBP or DBP to assess the response of BP to exercise. In the past decade, MAP, an index of overall BP, has been well established as a marker of cardiovascular risk in different clinical settings. ${ }^{28-30} \mathrm{BP}$ is usually characterized by its pulsatile and steady components. The steady component can be estimated by MAP and represents the left ventricular contractility, pulse rate and vascular resistance and elasticity averaged over time. ${ }^{31}$ Structural modifications of the peripheral small arteries are strongly associated with hypertension and have traditionally been considered to be responsible for high MAP. In fact, several studies have shown MAP to be closely associated with the progression of arterial stiffness ${ }^{32-34}$ and to be a dominant determinant, among a variety of BP parameters, of carotid atherosclerosis. ${ }^{35}$ For these reasons, we believe that the use of MAP as a measure of the response of BP to exercise enables us to detect more precisely the impacts of atherosclerotic risk factors on the vascular reactivity to increasing physical stress.

As has been noted by several authors, ${ }^{5}$ physical capacity may influence the effect of exercise on BP because maximum exercise capacity varies among individuals. The problem with using the $\mathrm{BP}$ readings at a fixed workload as a measure of the response of $\mathrm{BP}$ is that individuals will be performing at different percentages of their maximum capacities. This means that some adjustments are required for the differences in the subjects' physical exercise intensities at the workload at which the response of BP is determined. Therefore, in this study we evaluated the response of $\mathrm{BP}$ to exercise based on the relative increase in HR in response to workload. ${ }^{21}$ This approach can allow the comparison of individuals with varying levels of physical capacity; therefore, it may help to decrease the misidentification of exaggerated response of BP to exercise and more clearly identify the association of this phenomenon with the risk of hypertensive complications.
The current study has limitations that must be addressed. First, this was a cross-sectional observational study; therefore, causation cannot be determined for any of the observed relationships. The study sample was rather selective, consisting of middle-aged normotensive men without clinically manifest disease; this limits our ability to generalize the findings to women, younger or older persons, or patients with various disease statuses. Second, although we believe that vascular dysfunction and increased arterial stiffness may be responsible for the link between an excessive increase in MAP during dynamic exercise and the accumulation of MS components, we did not directly assess vascular functional and structural parameters in patients with an exaggerated response of $\mathrm{BP}$ to exercise. Third, in this study, we focused especially on vascular dysfunction as the causal factor for excessive increase in BP during exercise. However, the accumulation of metabolic risk factors also increases sympathetic activation. An exaggerated response of BP to exercise has been postulated to be related to excess stimulation of the sympathetic nervous system. ${ }^{36}$ Fourth, our study assessed the response of BP to exercise using the MAP during submaximal exercise. However, the MAP was not directly measured but was calculated from the formula $\mathrm{DBP}+(\mathrm{SBP}-\mathrm{DBP}) / 3$. In this regards, indirect measurements yield DBP readings that differ significantly from direct intravascular measurements during bicycle ergometer exercise at high intensity because such exercise requires isometric muscle contraction to a greater extent than low-intensity exercise. ${ }^{36}$ However, the indirect and direct measurements of DBP have been demonstrated to correlate satisfactorily below the workload of mild or moderate submaximal exercise. ${ }^{37}$

In conclusion, the present study has demonstrated that even in the prehypertensive state, combinations of MS components are significant correlates of an excessive increase in BP during dynamic exercise. These findings may be associated with the growing consensus that pathophysiological alterations of the vascular wall may contribute to the exaggerated response of $\mathrm{BP}$ to physical exertion. A rise in $\mathrm{BP}$ during exercise provides information about the cardiovascular reactivity to increasing physical stress that is not available from the BP at rest. Subclinical vascular impairment may affect vascular reactivity to a greater degree than basal vascular tone and hence could potentially be identified by an increased response of BP to exercise before the resting $\mathrm{BP}$ becomes elevated. The use of exercise testing solely for measuring exercise BP response is not recommended. However, it is possible to obtain exercise test results because the test is performed routinely to evaluate cardiorespiratory capacity in sports and occupational medicine as well as to detect coronary heart disease in hospital or office settings. Although more clinical studies are needed, the present findings suggest that monitoring of BP during exercise stress testing adds to the prognostic value of resting BP and may be a useful tool for risk stratification of individuals without clinically manifest disease.

\section{CONFLICT OF INTEREST}

The authors declare no conflict of interest.

\section{ACKNOWLEDGEMENTS}

This work was supported by a research grant from the Ministry of Education, Culture, Sports, Science and Technology of Japan (grant No. 12770201), by a grant from Chiyoda Mutual Life Foundation, Tokyo, Japan, by a grant from the Descent and Ishimoto Memorial Foundation, Osaka, Japan and by a grant from Wakayama Medical Award for young researchers of Wakayama Medical University. 
1 Singh JP, Larson MG, Manolio TA, O'Donnell CJ, Lauer M, Evans JC, Levy D. Blood pressure response during treadmill testing as a risk factor for new-onset hypertension. The Framingham heart study. Circulation 1999; 99: 1831-1836.

2 Sharabi Y, Ben-Cnaan R, Hanin A, Martonovitch G, Grossman E. The significance of hypertensive response to exercise as a predictor of hypertension and cardiovascular disease. J Hum Hypertens 2001; 15: 353-356.

3 Miyai N, Arita M, Miyashita K, Morioka I, Shiraishi T, Nishio I. Blood pressure response to heart rate during exercise test and risk of future hypertension. Hypertension 2002; 39: 761-766.

4 Lauer MS, Levy D, Anderson KM, Plehn JF. Is there a relationship between exercise systolic blood pressure response and left ventricular mass? The Framingham Heart Study. Ann Intern Med 1992; 116: 203-210.

5 Kurl S, Laukkanen JA, Rauramaa R, Lakka TA, Sivenius J, Salonen JT. Systolic blood pressure response to exercise stress test and risk of stroke. Stroke 2001; 32 : 2036-2041.

6 Mundal R, Kjeldsen SE, Sandvik L, Erikssen G, Thaulow E, Erikssen J. Exercise blood pressure predicts cardiovascular mortality in middle-aged men. Hypertension 1994; 24: 556-562

7 Weiss SA, Blumenthal RS, Sharrett AR, Redberg RF, Mora S. Exercise blood pressure and future cardiovascular death in asymptomatic individuals. Circulation 2010; 121: 2109-2116.

8 Stewart KJ, Sung J, Silber HA, Fleg JL, Kelemen MD, Turner KL, Bacher AC, Dobrosielski DA, DeRegis JR, Shapiro EP, Ouyang P. Exaggerated exercise blood pressure is related to impaired endothelial vasodilator function. Am J Hypertens 2004; 17: 314-320.

9 Olson KM, Augeri AL, Seip RL, Tsongalis GJ, Thompson PD, Pescatello LS. Correlates of endothelial function and the peak systolic blood pressure response to a graded maximal exercise test. Atherosclerosis. 2012; 222: 202-207.

10 Thanassoulis G, Lyass A, Benjamin EJ, Larson MG, Vita JA, Levy D, Hamburg NM, Widlansky ME, O'Donnell CJ, Mitchell GF, Vasan RS. Relations of exercise blood pressure response to cardiovascular risk factors and vascular function in the Framingham Heart Study. Circulation 2012; 125: 2836-2843.

11 Tsioufis C, Dimitriadis K, Thomopoulos C, Tsiachris D, Selima M, Stefanadi E, Tousoulis D, Kallikazaros I, Stefanadis C. Exercise blood pressure response, albuminuria, and arterial stiffness in hypertension. Am J Med 2008; 121: 894-902.

12 Tsiachris D, Tsioufis C, Dimitriadis K, Kokkinos P, Faselis C, Tousoulis D, Michaelides A, Papademetriou V. Stefanadis C. Relationship of ambulatory arterial stiffness index with blood pressure response to exercise in the early stages of hypertension. Blood Press Monit 2010; 15: 132-138.

13 Jae SY, Fernhall B, Heffernan KS, Kang M, Lee MK, Choi YH, Hong KP, Ahn ES, Park WH. Exaggerated blood pressure response to exercise is associated with carotid atherosclerosis in apparently healthy men. J Hypertens 2006; 24: 881-887.

14 Palatini P. Exaggerated blood pressure response to exercise: pathophysiologic mechanisms and clinical relevance. J Sports Med Phys Fitness 1998; 38: 1-9.

15 Mottillo S, Filion KB, Genest J, Joseph L, Pilote L, Poirier P, Rinfret S, Schiffrin EL, Eisenberg MJ. The metabolic syndrome and cardiovascular risk a systematic review and meta-analysis. J Am Coll Cardiol 2010; 56: 1113-1132.

16 Brett SE, Ritter JM, Chowienczyk PJ. Diastolic blood pressure changes during exercise positively correlate with serum cholesterol and insulin resistance. Circulation 2000; 101: 611-615.

17 Tsioufis C, Kasiakogias A, Tsiachris D, Kordalis A, Thomopoulos C, Giakoumis M, Bounas P, Pittaras A, Michaelides A, Stefanadis C. Metabolic syndrome and exaggerated blood pressure response to exercise in newly diagnosed hypertensive patients. Eur J Prev Cardiol 2012; 19: 467-473.
18 de Lima SG, de Albuquerque MD, de Oliveira JR, Ayres CF, da Cunha JE, de Oliveira DF, de Lemos RR, de Souza MB, E Silva OB. Exaggerated blood pressure response during exercise treadmill testing: functional and hemodynamic features, and risk factors. Hypertens Res 2012; 35: 733-8.

19 Papavasileiou MV, Thomopoulos C, Antoniou I, Papadimitriou G, Seferou M, Makris TK. Impaired glucose metabolism and the exaggerated blood pressure response to exercise treadmill testing in normotensive patients. J Clin Hypertens 2009; 11: 627-635.

20 Lightfoot JT, Tankersley C, Rowe SA, Freed AN, Fortney SM. Automated blood pressure measurements during exercise. Med Sci Sports Exerc 1989; 21: 698-707.

21 Karvonen J, Vuorimaa T. Heart rate and exercise intensity during sports activities. Practical application. Sports Med 1988; 5: 303-311.

22 Examination Committee of Criteria for the Metabolic Syndrome in Japan. Definition and criterion of metabolic syndrome. Jpn Soc Intern Med 2005; 94: 188-203 (in Japanese).

23 Gilligan DM, Panza JA, Kilcoyne CM, Waclawiw MA, Casino PR, Quyyumi AA. Contribution of endothelium-derived nitric oxide to exercise-induced vasodilation. Circulation 1994; 90: 2853-2858.

24 Daida H, Allison TG, Squires RW, Miller TD, Gau GT. Peak exercise blood pressure stratified by age and gender in apparently healthy subjects. Mayo Clin Proc 1996; 71: 445-452.

25 Mackey RH, Sutton-Tyrrell K, Vaitkevicius PV, Sakkinen PA, Lyles MF, Spurgeon HA, Lakatta EG, Kuller LH. Correlates of aortic stiffness in elderly individuals: a subgroup of the Cardiovascular Health Study. Am J Hypertens 2002; 15: 16-23.

26 Mazzeo RS, Marshall P. Influence of plasma catecholamines on the lactate threshold during graded exercise. J Appl Physiol 1989; 67: 1319-1322.

27 Maeda S, Miyauchi T, Goto K, Mastuda M. Alteration of plasma endthelin-1 by exercise at intensities lower and higher than ventilatory threshold. J Appl Physiol 1994; 77: 1399-1402.

28 Safar ME, Boudier HS. Vascular development, pulse pressure, and the mechanisms of hypertension. Hypertension 2005; 46: 205-209.

29 Zheng L, Sun Z, Li J, Zhang R, Zhang X, Liu S, Li J, Xu C, Hu D, Sun Y. Pulse pressure and mean arterial pressure in relation to ischemic stroke among patients with uncontrolled hypertension in rural areas of China. Stroke 2008; 39: 1932-1937.

30 Millar JA, Lever AF, Burke V. Pulse pressure as a risk factor for cardiovascular events in the MRC Mild Hypertension Trial. J Hypertens 1999; 17: 1065-1072.

31 Benetos A, Laurent S, Asmar RG, Lacolley P. Large artery stiffness in hypertension. J Hypertens 1997; 15 (Suppl): S89-S97.

32 Payne RA, Wilkinson IB, Webb DJ. Arterial stiffness and hypertension: emerging concepts. Hypertension 2010; 55: 9-14.

33 Cheng LT, Chen HM, Tang LJ, Tang W, Huang HY, Gu Y, Wang T. The study of aortic stiffness in different hypertension subtypes in dialysis patients. Hypertens Res 2008 . 31: 593-599.

34 Gunarathne A, Patel JV, Gammon B, Hughes EA, Lip GY. Impact of mean arterial blood pressure on higher arterial stiffness indices in South Asians compared to white Europeans. J Hypertens 2008; 26: 1420-1426.

35 Yilmaz MI, Qureshi AR, Carrero JJ, Saglam M, Suliman ME, Caglar K, Eyileten T, Sonmez A, Oguz Y, Vural A, Yenicesu M, Axelsson J. Predictors of carotid artery intimamedia thickness in chronic kidney disease and kidney transplant patients without overt cardiovascular disease. Am J Nephrol 2010; 31: 214-221.

36 Wilson MF, Sung BH, Pincomb GA, Lovallo WR. Exaggerated pressure response to exercise in men at risk for systemic hypertension. Am J Cardiol 1990; 66: 731-736

37 Griffin SE, Robergs RA, Heyward VH. Blood pressure measurement during exercise: a review. Med Sci Sports Exerc 1997; 29: 149-159. 